\section{Inversionsschichten in Silicium an der Grenze zu einem Elektrolyten ${ }^{1}$}

\author{
Von H.-U. HARTEN
}

Philips-Zentrallaboratorium Hamburg

(Z. Naturforschg. 16 a, 1401 [1961] ; eingegangen am 30. November 1961)

Unter der Oberfläche eines Halbleiters befindet sich normalerweise eine Raumladungsrandschicht; sie wird charakterisiert durch die Bandaufwölbung bzw. das Oberflächenpotential $\varphi_{\mathrm{S}}\left(e \varphi_{\mathrm{S}}\right.$ ist definiert als der $\mathrm{Ab}$ stand der FERMI-Energie von der Mitte der verbotenen Zone an der Oberfläche des Halbleiters). Es ist üblich, zur Bestimmung von $\varphi_{\mathrm{s}}$ die Oberflächenleitfähigkeit $\Delta G$ zu messen, die man aus dem Leitwert der untersuchten Probe parallel zu ihrer Oberfläche erhält. $\Delta G$ ist hoch bei einer Anreicherungsrandschicht (hohe Majoritätskonzentration), nimmt ab im Verarmungsgebiet und steigt wieder an, wenn sich eine Inversionsschicht mit hoher Konzentration der Minoritätsträger ausbildet. Der genaue Zusammenhang zwischen $\Delta G$ und $\varphi_{\mathrm{s}}$ ist im Prinzip bekannt ${ }^{2}$.

Wie frühere Untersuchungen gezeigt haben ${ }^{3}$, kann man bei Silicium die Oberflächenleitung auch dann messen, wenn sich die Probe in einem Elektrolyten befindet. Bei diesen Versuchen war das Oberflächenpotentiul durch eine von außen zwischen Silicium und Elekt $\neq$ olyt angelegte Spannung variiert worden. Infolgedessen floß ein Strom senkrecht zur Oberfläche, der unter Umständen das thermodynamische Gleichgewicht der Trägerkonzentrationen in der Randschicht störte. Bei hinreichend hoher Belastung in Sperrichtung verhielt sich die Randschicht im allgemeinen ebenso wie die Sperrschicht eines p-n-Überganges: Sie verarmte sowohl an Elektronen wie auch an Löchern. Daher bildete sich keine Inversionsschicht aus und die Oberflächenleitung zeigte darum auch kein Minimum.

Um eine Inversionsschicht beobachten zu können, muß man demnach das Oberflächenpotential variieren, ohne einen Brutto-Strom senkrecht zur Oberfläche hervorzurufen. Das läßt sich durch eine Änderung der Zusammensetzung des Elektrolyten erreichen, die das Galvani-Potential an der Elektrode beeinflußt.

Abb. 1 zeigt das Ergebnis entsprechender Messungen an einer n-leitenden und einer p-leitenden SiliciumProbe. (Die experimentelle Anordnung entspricht weitgehend der früherer Versuche ${ }^{3}$.) Als Elektrolyt dienten Lösungen von Cer(IV)ammoniumsulfat in $1-n$. $\mathrm{H}_{2} \mathrm{SO}_{4}$, und zwar in molaren Konzentrationen zwischen $10^{-2}$ und $3 \cdot 10^{-5}$. Die Meßpunkte der Oberflächenleitung sind auf die Spannung $U_{\mathrm{K}}$ bezogen, die zwischen einem sperrfreien Kontakt am Silicium und einer Kalomel-Elektrode im Elektrolyten auftrat. Sie lassen deutlich das erwartete Minimum der Oberflächenleitung erkennen. Die ausgezogenen Kurven sind für das verwendete Silicium berechnet und beziehen sich auf das Oberflächenpotential als $\varphi_{\mathrm{s}}$. Die Skalen für $U_{\mathrm{K}}$ und $\varphi_{\mathrm{s}}$ haben gleichen Maßstab, sind aber gegeneinander ver-

1 Teilweise vorgetragen auf der Physikertagung in Bad Pyrmont; Phys. Verh. 1961, 27. schoben. Da sich die Meßpunkte gut an die berechneten Kurven anpassen, müssen sich $U_{\mathrm{K}}$ und $\varphi_{\mathrm{s}}$ jeweils um den gleichen Betrag geändert haben. Bei den vorliegenden Messungen sind also alle Änderungen der GalvaniSpannung zwischen Silicium und Elektrolyt nur auf Änderungen der Raumladungsrandschicht im Silicium zurückzuführen.

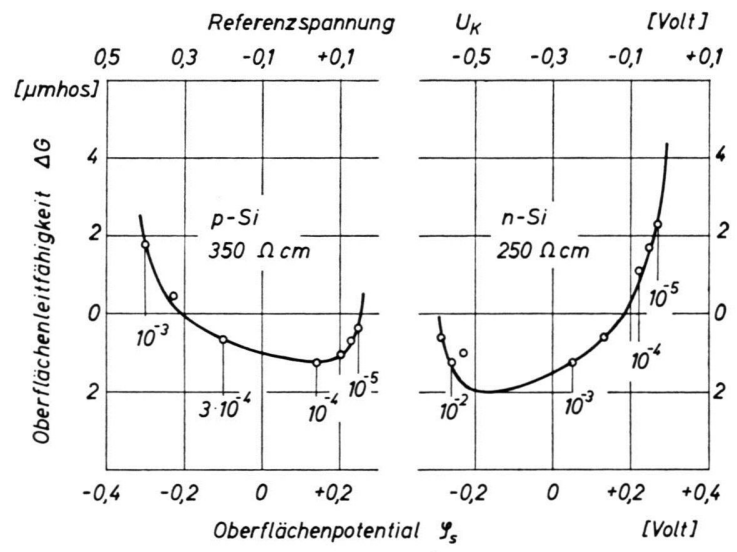

Abb. 1. Oberflächenleitfähigkeit $\Delta G$ von zwei Si-Proben in Abhängigkeit von der Referenzspannung $U_{\mathrm{K}}$ der KalomelElektrode (Meßpunkte) und dem Oberflächenpotential $\varphi_{\mathrm{S}}$ (berechnete Kurve). Elektrolyt: Cer(IV)ammoniumsulfat in l- $n$. $\mathrm{H}_{2} \mathrm{SO}_{4}$; die Zahlen an den Meßpunkten bezeichnen die molare Konzentration des Sulfats.

Durch die verwendeten Elektrolyte wurde nicht immer der in der Abbildung gezeigte Bereich des Oberflächenpotentials überdeckt. Blieb die Probe längere Zeit in einer der Lösungen stehen, so verschoben sich $\varphi_{\mathrm{s}}$ und $U_{\mathrm{K}}$ irreversibel. Wechsel der Elektrolyten hatte dann zwar wieder reversible Änderungen beider Größen zur Folge, der einfache Zusammenhang zwischen ihnen blieb jedoch nicht immer erhalten. Aus diesem Grunde wurden die Proben vor jeder Meßreihe geätzt $(\mathrm{HF}+$ $\left.\mathrm{HNO}_{3}+\mathrm{H}_{2} \mathrm{O}\right)$.

Bei p-Silicium trat die Inversion immer auf, wenn das Oberflächenpotential in den entsprechenden Bereich gebracht werden konnte. Bei n-Silicium ließ sich das Minimum der Oberflächenleitung jedoch oft nur dann sicher beobachten, wenn die Oberfläche wenigstens an einer Stelle mechanisch beschädigt worden war. Das dürfte darauf zurückzuführen sein, daß die potentialbestimmende Reaktion Löcher verbraucht, die u. U. nur dann in genügender $\mathrm{Zahl}$ verfügbar sind, wenn zumindest in einem begrenzten Gebiet hohe Oberflächenrekombinationen und damit -generation herrscht.

Untersuchungen dieser Art wurden angeregt durch Herrn Dr. H. J. G. VINK, bei der Auswahl der Elektrolyten beriet Herr Dr. F. W. Dorn. Die experimentellen Arbeiten führte in zahlreichen Vorversuchen Frl. I. Moser, bei den abschließenden Messungen Frl. G. BRAUER durch. Ich danke allen herzlich.

2 V. O. Mowery, J. Appl. Phys. 29, 1753 [1958].

3 H. U. Harten, Z. Naturforschg. 16 a, 459 [1961]. 